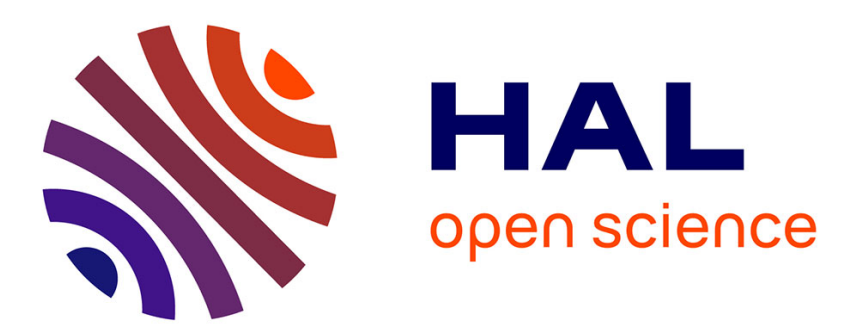

\title{
Free Energy Profiles along Consensus Normal Modes Provide Insight into HIV-1 Protease Flap Opening
}

Paulo R Batista, Gaurav Pandey, Pedro G Pascutti, Paulo M Bisch, David

Perahia, Charles Robert

\section{- To cite this version:}

Paulo R Batista, Gaurav Pandey, Pedro G Pascutti, Paulo M Bisch, David Perahia, et al.. Free Energy Profiles along Consensus Normal Modes Provide Insight into HIV-1 Protease Flap Opening. Journal of Chemical Theory and Computation, 2011, 7 (8), pp.2348-2352. 10.1021/ct200237u . hal-02988887

\section{HAL Id: hal-02988887 https://hal.science/hal-02988887}

Submitted on 4 Nov 2020

HAL is a multi-disciplinary open access archive for the deposit and dissemination of scientific research documents, whether they are published or not. The documents may come from teaching and research institutions in France or abroad, or from public or private research centers.
L'archive ouverte pluridisciplinaire $\mathbf{H A L}$, est destinée au dépôt et à la diffusion de documents scientifiques de niveau recherche, publiés ou non, émanant des établissements d'enseignement et de recherche français ou étrangers, des laboratoires publics ou privés. 


\title{
Free Energy Profiles along Consensus Normal Modes Provide Insight into HIV-1 Protease Flap Opening
}

\author{
Paulo R. Batista, ${ }^{+, \S, \perp}$ Gaurav Pandey, ${ }^{\S, \neq}$ Pedro G. Pascutti, ${ }^{+}$Paulo M. Bisch, ${ }^{\dagger}$ David Perahia, ${ }^{*, \perp, \S, \#}$ and \\ Charles H. Robert ${ }^{\S, \|, \#}$ \\ ${ }^{\dagger}$ Instituto de Biofísica Carlos Chagas Filho, Universidade Federal do Rio de Janeiro, 21941-902, Brasil \\ ${ }^{\ddagger}$ Indian Institute of Technology, Roorkee, 247667, India \\ ${ }^{\S}$ CNRS Institut de Biochimie et Biophysique Moléculaire et Cellulaire, Université Paris Sud 11, 91405 Orsay, France \\ "CNRS Laboratoire de Biochimie Théorique, Institut de Biologie Physico Chimique, Université Paris Diderot, Sorbonne Paris Cité, \\ 75005 Paris, France \\ ${ }^{\perp}$ CNRS BIMoDyM -Laboratoire de Biologie et Pharmacologie Appliquées - École Normale Supérieure de Cachan, 94235 Cachan, France
}

Supporting Information

ABSTRACT: Describing biological macromolecular energetics from computer simulations can pose major challenges, and often necessitates enhanced conformational sampling. We describe the calculation of conformational free-energy profiles along carefully chosen collective coordinates: "consensus" normal modes, developed recently as robust alternatives to conventional normal modes. In an application to the HIV-1 protease, we obtain efficient sampling of significant flap opening movements governing inhibitor binding from relatively short simulations, in close correspondence with experimental results.

$P_{t}$ redicting protein dynamics remains a challenge in computational biology. The configurational space in which a solvated macromolecule evolves is so large that we cannot reasonably expect to ever explore it fully. Standard molecular dynamics (MD) simulations have their limits: for proteins of normal size, all-atom simulations are typically limited to time scales largely inferior to that of many biologically interesting movements and conformational changes. Although such limits to computation are being pushed back every year (e.g., ref 1), exploration of long-timescale dynamics by MD alone is still not feasible. The difficulty is especially acute for the identification of new molecules binding flexible biological targets and understanding related allosteric effects. ${ }^{2,3}$ Improved understanding of the dynamics of biological macromolecules can thus facilitate the proposal of new inhibitors or modulators of biological macromolecules in the search for new therapies. ${ }^{4,5}$

Properly taking into account features of the energy landscape "in the large" allows one to arrive at accurate conclusions concerning the dynamic behavior of macromolecules while using shorter simulations. For example, coarse-grained potentials represent groups of atoms by a single, larger particle, leading to increased efficiency of the simulation. ${ }^{6,7}$ Other approaches are based on the use of biasing techniques to enhance MD sampling along interesting directions in the conformational space, using for example umbrella sampling or metadynamics..$^{8-12}$ In both cases, the goal is essentially to look past noisier, smaller-scale degrees of freedom in order to exploit larger-scale features of the structure and/ or the energy surface. Representative collective-coordinate descriptions must be found that are appropriate to the system under study. Principal components obtained from molecular simulations of the macromolecule have been successfully adapted for this purpose. ${ }^{13-15}$ Normal modes analysis of the macromolecular structure also provides information concerning its most favorable modes of deformation, which correspond to gently sloped directions on the energy landscape. ${ }^{16-18}$ Normal-mode-based coordinates have been correlated with directions of conformational change in known systems ${ }^{19-21}$ and offer productive directions for exploring the mechanisms of conformational change. ${ }^{22,23}$ Consensus normal modes take this one step further through a statistical approach to identifying robust directions from multiple normal mode descriptions, in which the shape of the free energy surface is averaged over several minima via the covariance matrix. ${ }^{24}$ As well as being more robust to intrinsic variability in the analyzed structure, the resulting consensus modes are more collective than the individual normal modes and provide a more natural means of accommodating solvent effects. ${ }^{24}$

A particularly challenging case is that of the HIV-1 protease (PR), the major target of anti-AIDS drugs. PR is a homodimer consisting of two chains of 99 amino acid residues in which access to a central substrate binding site is mediated by symmetrically disposed $\beta$ hairpins called the "flaps". ${ }^{25}$ Movement of the flaps has been shown in experimental studies to be critical for inhibitor binding. ${ }^{25}$ Indeed, 450 crystal or NMR structures of this molecule under various conditions show conformational variability mainly in this region (Figure S1, Supporting Information). Interflap distance distributions established by electron paramagnetic resonance (EPR) measurements using spin labeling confirm the importance of flap conformational dynamics in solution. ${ }^{26}$ Characterizing these dynamics is thus critical in the search for new inhibitors of the protease and for understanding mutations that allow HIV to evade existing therapies. ${ }^{27}$

Received: April 7, 2011

Published: July 01, 2011 

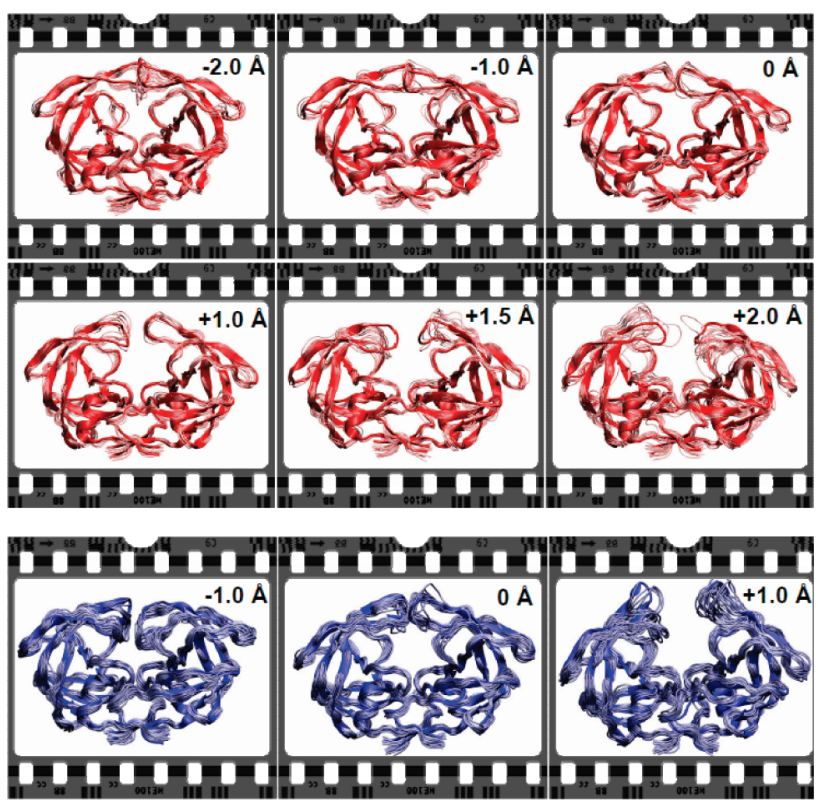

$\square 3^{\text {rd }} \mathrm{CM}$

$5^{\text {th }} \mathrm{CM}$

Figure 1. Snapshots of HIV-1 protease taken from MD simulations with umbrella sampling along the consensus mode directions describing flap opening. Each frame contains 20 superposed snapshots sampled at 5 ps intervals from an independent $\mathrm{MD}$ trajectory restrained to a particular normal mode value: from -2 to $+2 \AA$ for the mode 3 and from -1 to $+1 \AA$ for mode 5 .

Nevertheless, the amplitude of the conformational dynamics seen in unbiased MD simulations only accounts for a small part of the conformational variability observed experimentally, due to the long time scales characterizing the motion, which have been estimated to be on the order of several hundred nanoseconds or microseconds. ${ }^{28,29}$ Further, enhanced sampling methods do not always increase exploration of flap opening movements. In a recent study of the binding of a peptide substrate to PR, seven different collective coordinates were biased simultaneously using explicit solvent simulations over $1.6 \mu \mathrm{s} .{ }^{30}$ Only semiopen structures were observed; full flap opening that would be necessary to allow the entrance of the natural polyprotein substrate was not observed.

We show here that umbrella sampling along strongly collective consensus modes of the HIV-1 protease permits calculation of a potential of mean force (PMF) along these reaction coordinates using relatively short $\mathrm{MD}$ simulations. The collective coordinates allow statistical sampling of representative conformations of the protein with alternative flap conformations including highly open states and largely account for the conformational dynamics observed in the spin label measurements.

The consensus modes (CM) for the apo HIV-1 protease were calculated as described previously from normal modes analyses of 20 protease structures, which were extracted at regular intervals from a $1 \mathrm{~ns}$ explicit-solvent MD simulation and quenched. ${ }^{24} \mathrm{An}$ advantage of the consensus modes formalism is its adaptability to practical constraints; here, for example, the consensus modes were calculated for the $\alpha$ carbons alone in order to obtain applicability to mutational studies, yet they reflect a set of PR conformations sampled from the original all-atom, explicit solvent MD simulation as well as the individual hydration layers used in the initial normal mode determinations. ${ }^{24}$ The third and fifth lowest consensus modes most involved in flap movement are shown in Figure 1. In the orientation shown, mode 3 is associated with a horizontal separation of the flaps while mode 5 describes vertical movement of the flaps, which expands the active site. A movie of the individual mode movements is provided (see Supporting Information, movies 1 and 2).

Sampling along the consensus mode directions was performed using generalized-Born implicit solvent ${ }^{31} \mathrm{MD}$ simulations, using the VMOD command integrated into CHARMM version $\mathrm{c} 355^{32}$ This command adds a term to the energy function which is proportional to the squared deviation of the instantaneous normal coordinate of the protein from a prescribed target value. In this procedure, a given instantaneous structure $\mathbf{R}$ is first superimposed onto a structure $\mathbf{R}_{\mathbf{o}}$ that served as the reference for the consensus modes calculations. The normal coordinate is then defined as the projection of the mass-weighted coordinate difference vector between $\mathbf{R}$ and the $\mathbf{R}_{\mathbf{o}}$ onto the mode vector considered. VMOD can be used to bias several collective coordinates simultaneously. The approach has been used along with energy minimization to explore the effects of variability in the shape of the binding pocket in inhibitor docking to proteins $s^{33,34}$ and to the study of the energy variations of large conformational changes. ${ }^{23,35}$ In the current study, we used 41 independent $\mathrm{MD}$ simulations, each restrained by VMOD to a different consensus normal mode coordinate (from -2 to $+2 \AA$, every $0.1 \AA$ ). The starting structure was first displaced to the target coordinate by energy minimization using the VMOD restraint; MD heating, equilibration, and production followed, all performed with the restraint in place in order to obtain the desired sampling. PMFs along the consensus mode coordinate were calculated using the weighted histogram (WHAM) procedure as implemented by Grossfield. ${ }^{36}$ Additional details are given in the Supporting Information and associated Figure S2. We emphasize that exploring a normal mode coordinate using a VMOD restraint is not the same as simply displacing the structure along the normal coordinate. Employment of VMOD restricts only the normal mode coordinate itself; the remaining degrees of freedom are free to adapt to the imposed coordinate restraint.

The PMFs calculated for consensus modes 3 and 5 are shown in Figure 2. The free energy profile along mode 3 (top panel) shows a wide basin for displacements ranging from $-1.5 \AA$ to $+1.5 \AA$ A with energy less than $2 k T$, while mode 5 (bottom panel) shows a narrower basin between -0.5 and $+0.5 \AA$. In both plots, the sign has been chosen so that the negative coordinate corresponds to closure of the flaps and the positive to opening. Outside the basin region, the free energy increases rapidly. We also show in red the distributions of normal mode coordinates calculated for the 450 known PDB structures, as described in the Supporting Information. Most of these structures contain ligands and are thus of the closed form, and correspondingly the bulk of the distribution for each mode is seen to lie to the left of the origin. However, several unbound forms of the protease have been solved as well, and these correspond to the small peaks around $0.5 \AA$ in the distribution of mode 3 . For both open and closed forms, the experimentally determined structures are seen to represent low-energy regions of the PMFs, as should be expected. In comparing the PMFs of modes 3 and 5, we see that the profile along mode 5, in which the flaps move vertically in Figure 1, is higher in energy but simpler in form than that of mode 3 , associated with horizontal flap separation. As can be seen in Figure 2, mode 3 shows small barriers on the order of $2 k T$ which tend to delineate the starting structure from either more-open or moreclosed forms. 


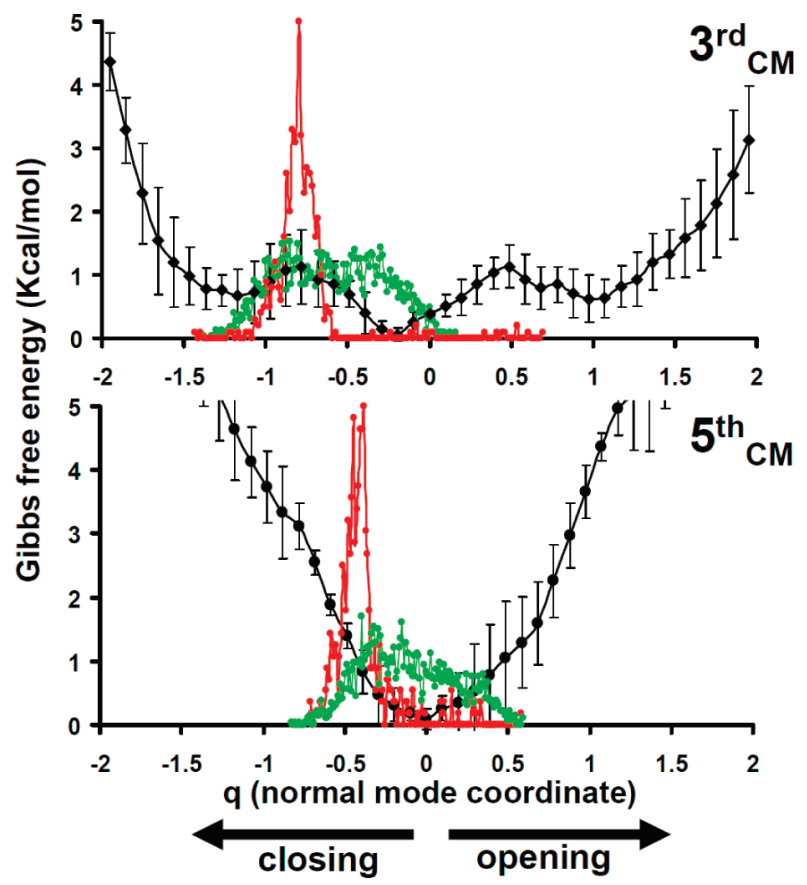

Figure 2. Free energy profiles for PR flap opening motions along CM directions. Two different modes (3rd and 5th CMs) were explored. The black line corresponds to the mean $( \pm S D)$ of the free energies obtained over three independent simulations. The distribution in red shows the normal mode coordinates calculated for the 450 experimental PR structures deposited in the Protein Data Bank. The distribution shown in green corresponds to the normal mode coordinates of structures sampled from an unrestrained $50 \mathrm{~ns}$ explicit-solvent $\mathrm{MD}$ simulation.

The necessity for umbrella restraints in exploring the flap opening can be seen by examining the normal mode coordinates calculated for the structures obtained from a 50 ns unrestrained MD simulations, shown in the distribution in green (Figure 2). Clearly, the unrestrained MD sampled closed forms of the binding site rather well, producing structures having projections along both modes 3 and 5 that are consistent with the calculated PMFs. But the sampling of open forms along consensus mode 3 was poor. This may be related to the barrier seen at $0.5 \AA$ in Figure 2 (top) for this mode, although this barrier must be of a different nature from that at $-0.75 \AA$ of the same height, which did not prevent sampling in the direction of closing. The asymmetry of the sampling seen in the unrestrained MD simulations in this study is consistent with the under-representation of highly open structures seen in over 460 replicated 50 ns explicit solvent MD simulations reported by Sadiq and De Fabritiis, ${ }^{37}$ in which it was suggested that the time scale for the conformational dynamics accompanying full flap opening is very long and judging from NMR studies may be on the order of microseconds and beyond. ${ }^{28,37}$

Another way of looking at flap opening is to calculate the distance between the flaps. In $\mathrm{EPR}^{26}$ and $\mathrm{MD}^{38}$ studies of the protease, spin labels attached to residue 55 in each flap allowed determination of the interflap distance distribution in the presence and absence of the protease inhibitor ritonavir. Figure 3 compares the experimental distribution for the apo-protease (in red) to the distribution of distances calculated for structures obtained from a $50 \mathrm{~ns}$ explicit solvent unrestrained MD simulation (green). It is clear that the unrestrained simulations do not sample structures having the larger interflap distances seen in the EPR results.

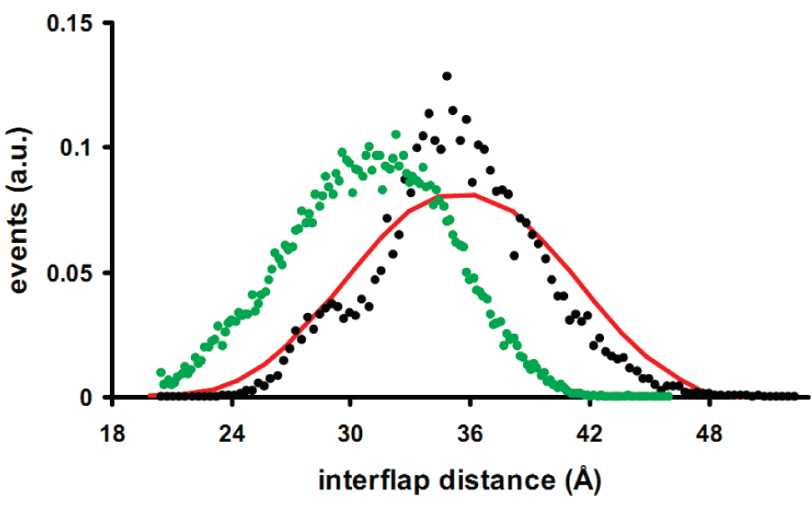

Figure 3. Correspondence between interflap distances (using Lys55 $\mathrm{NZ}$ atoms) obtained from MD with umbrella sampling (black) and from EPR of the spin-labeled protease (red). Data for structures extracted from an unrestrained explicit-solvent $50 \mathrm{~ns} \mathrm{MD}$ are also shown (green).

Figure 3 also shows the distance distribution obtained for structures sampled in the MD simulations employing umbrella sampling along consensus mode 3 (in black), in which each distance has been weighted by the Boltzmann factor corresponding to the PMF shown in Figure 2 in order to allow comparison. The agreement with the experimental curve suggests that both closed and open flap conformations are properly sampled along this reduced coordinate.

Sutto et al., in comparing unbiased and biased exploration of the energy surface for a pentapeptide, concluded that the sampling performance strongly depends on the choice of the collective coordinates. ${ }^{15}$ Indeed, other collective coordinates used for the HIV-1 protease did not particularly enhance sampling of large flap opening movements, although they were sufficient to study peptide binding. ${ }^{30}$ From this point of view, the consensus modes provide valuable collective coordinates for exploring the HIV-1 PR potential energy surface in the direction of flap opening, as will be necessary for characterizing conformational factors playing a role in the binding of the natural substrate.

Several conclusions can be drawn from this study, both for consensus normal mode sampling in general and for the dynamics of the HIV-1 protease. First, the use of umbrella sampling along a well-chosen collective coordinate-here, consensus mode directions - enables one to explore HIV-1 protease flap opening that would require long conventional $\mathrm{MD}$ simulations in order to properly account for its conformational and energetic aspects. The free energy increase associated with highly open structures is less than $2 k T$ despite their rarity in conventional simulations, underlining the kinetic difficulties that can confront sampling using conventional MD. Also, the distribution of experimental PR structures, the majority containing ligands, on the free energy landscape clearly shows that a population shift mechanism applies, ${ }^{39-42}$ as the binding of the ligand would appear to stabilize closed forms of the apoprotein that are already energetically allowed, in agreement with pulsed EPR experiments. ${ }^{43}$ It may also be noted that the free energy landscape along the opening/closing mode (Figure 2) is rather flat, with a highly anharmonic nature, while that corresponding to a higher energy mode (5th mode in Figure 2) is closer to a quadratic shape. The PMF approach using the consensus normal mode direction as the collective coordinate allows one to estimate upper bounds for the amplitude of large conformational changes and corresponding energy, and to describe structural aspects that may be very different from those derived from a simple normal-mode analysis itself. 
Further, using consensus modes ${ }^{24}$ allowed us to frame the problem of exploring the conformational space of the HIV-1 protease in terms of the $\alpha$ carbon atoms alone, while the mode determinations themselves still benefit from the high quality of the individual normal mode determinations in which an all-atom representation of the protein was used in addition to a solvation layer. ${ }^{44}$ We note that the protocol used here is entirely adaptable for use on distributed computing platforms, as the simulations corresponding to the individual displacements along the mode coordinate are completely independent. This will enable us to further explore the protease motions to understand the effects of certain mutants such as the drug-resistant MDR769, in which experimental evidence suggests the flap distribution to be shifted toward much larger distances. ${ }^{45}$

Improved free energy evaluations from MD simulations, which as we have shown here can be obtained using enhanced sampling along consensus normal mode directions, offers the possibility of differentiating the behavior of different mutants of HIV-1 in view of obtaining clues about their resistance mechanisms.

\section{ASSOCIATED CONTENT}

Supporting Information. Additional data, movies of the individual mode movements, and information regarding methods and protocols are included. This material is available free of charge via the Internet at http://pubs.acs.org

\section{AUTHOR INFORMATION}

\section{Corresponding Author}

*E-mail: david.perahia@lbpa.ens-cachan.fr, robert@ibpc.fr.

\section{Author Contributions}

${ }^{\#}$ D.P. and C.H.R. contributed equally to the conception and design of this study.

\section{ACKNOWLEDGMENT}

We wish to thank the Brazilian agencies CAPES, CNPq, and FAPERJ as well as the French-Brazilian CAPES/COFECUB collaboration project for financial support and the French Ministry of Foreign and European Affairs for supporting the participation of G.P. in this project. The University of Paris Orsay Pluriformation Project DEMAIN provided computing resources used in this study.

\section{REFERENCES}

(1) Shaw, D. E.; Maragakis, P.; Lindorff-Larsen, K.; Piana, S.; Dror, R. O.; Eastwood, M. P.; Bank, J. A.; Jumper, J. M.; Salmon, J. K.; Shan, Y.; Wriggers, W. Atomic-level characterization of the structural dynamics of proteins. Science 2010, 330 (6002), 341-6.

(2) Ahmed, A.; Kazemi, S.; Gohlke, H. Protein Flexibility and Mobility in Structure-Based Drug Design. Front. Drug Des. Discovery 2007, 3, $455-476$.

(3) Costa, M.; Batista, P.; Shida, C.; Robert, C.; Bisch, P.; Pascutti, P. How does heparin prevent the $\mathrm{pH}$ inactivation of cathepsin $\mathrm{B}$ ? Allosteric mechanism elucidated by docking and molecular dynamics. BMC Genomics 2010, 11 (Suppl 5), S5.

(4) B-Rao, C.; Subramanian, J.; Sharma, S. D. Managing protein flexibility in docking and its applications. Drug Discovery Today 2009, 14 (7-8), 394-400.

(5) Zwier, M. C.; Chong, L. T. Reaching biological timescales with all-atom molecular dynamics simulations. Curr. Opin. Pharmacol. 2010, 10 (6), 745-752.
(6) Levitt, M.; Warshel, A. Computer simulation of protein folding. Nature 1975, 253 (5494), 694-8.

(7) Clementi, C. Coarse-grained models of protein folding: toy models or predictive tools?. Curr. Opin. Struct. Biol. 2008, 18 (1), 10-15.

(8) Torrie, G. M.; Valleau, J. P. Nonphysical sampling distributions in Monte Carlo free-energy estimation: Umbrella sampling. J. Comput. Phys. 1977, 23 (2), 187-199.

(9) Dellago, C.; Bolhuis, P. G. Transition Path Sampling and Other Advanced Simulation Techniques for Rare Events. Adv. Comput. Simulat. Approaches Soft Matter Sci. III 2009, 221, 167-233.

(10) Spiwok, V.; Lipovova, P.; Kralova, B. Metadynamics in essential coordinates: free energy simulation of conformational changes. J. Phys. Chem. B 2007, 111 (12), 3073-6.

(11) Tribello, G. A.; Ceriotti, M.; Parrinello, M. A self-learning algorithm for biased molecular dynamics. Proc. Natl. Acad. Sci. U.S.A. 2010, 107 (41), 17509-17514.

(12) Autieri, E.; Sega, M.; Pederiva, F.; Guella, G. Puckering free energy of pyranoses: A NMR and metadynamics-umbrella sampling investigation. J. Chem. Phys. 2010, 133 (9), 095104.

(13) Spiwok, V.; Lipovova, P.; Kralova, B. Metadynamics in essential coordinates: Free energy simulation of conformational changes. J. Phys. Chem. B 2007, 111 (12), 3073-3076.

(14) Spiwok, V.; Kralova, B.; Tvaroska, I. Continuous metadynamics in essential coordinates as a tool for free energy modelling of conformational changes. J. Mol. Model. 2008, 14 (11), 995-1002.

(15) Sutto, L.; D'Abramo, M.; Gervasio, F. L. Comparing the Efficiency of Biased and Unbiased Molecular Dynamics in Reconstructing the Free Energy Landscape of Met-Enkephalin. J. Chem. Theory Comput. 2010, 6 (12), 3640-3646.

(16) Karplus, M.; Kushick, J. N. Method for estimating the configurational entropy of macromolecules. Macromolecules 1981, 14 (2), $325-332$.

(17) Levy, R. M.; Perahia, D.; Karplus, M. Molecular dynamics of an alpha-helical polypeptide: Temperature dependence and deviation from harmonic behavior. Proc. Natl. Acad. Sci. U.S.A. 1982, 79 (4), 13461350.

(18) Levy, R. M.; Karplus, M.; Kushick, J.; Perahia, D. Evaluation of the configurational entropy for proteins: application to molecular dynamics simulations of an alpha-helix. Macromolecules 1984, 17 (7), 1370-1374.

(19) Tama, F.; Sanejouand, Y. H. Conformational change of proteins arising from normal mode calculations. Protein Eng. 2001, 14 (1), $1-6$.

(20) Brooks, B.; Karplus, M. Normal modes for specific motions of macromolecules: application to the hinge-bending mode of lysozyme. Proc. Natl. Acad. Sci. U.S.A. 1985, 82 (15), 4995-9.

(21) Yang, Z.; Majek, P.; Bahar, I. Allosteric transitions of supramolecular systems explored by network models: application to chaperonin GroEL. PLoS Comput. Biol. 2009, 5 (4), e1000360.

(22) Zheng, W.; Brooks, B. R.; Hummer, G. Protein conformational transitions explored by mixed elastic network models. Proteins 2007, 69 (1), 43-57.

(23) Floquet, N.; Durand, P.; Maigret, B.; Badet, B.; Badet-Denisot, M. A.; Perahia, D. Collective motions in glucosamine-6-phosphate synthase: influence of ligand binding and role in ammonia channelling and opening of the fructose-6-phosphate binding site. J. Mol. Biol. 2009, 385 (2), 653-64.

(24) Batista, P. R.; Robert, C. H.; Marechal, J. D.; Hamida-Rebai, M. B.; Pascutti, P. G.; Bisch, P. M.; Perahia, D. Consensus modes, a robust description of protein collective motions from multiple-minima normal mode analysis--application to the HIV-1 protease. Phys. Chem. Chem. Phys. 2010, 12 (12), 2850-9.

(25) Gustchina, A.; Weber, I. T. Comparison of inhibitor binding in HIV-1 protease and in non-viral aspartic proteases: the role of the flap. FEBS Lett. 1990, 269 (1), 269-72.

(26) Galiano, L.; Bonora, M.; Fanucci, G. E. Interflap distances in HIV-1 protease determined by pulsed EPR measurements. J. Am. Chem. Soc. 2007, 129 (36), 11004-5. 
(27) Piana, S.; Carloni, P.; Rothlisberger, U. Drug resistance in HIV-1 protease: Flexibility-assisted mechanism of compensatory mutations. Protein Sci. 2002, 11 (10), 2393-402.

(28) Ishima, R.; Freedberg, D. I.; Wang, Y. X.; Louis, J. M.; Torchia, D. A. Flap opening and dimer-interface flexibility in the free and inhibitor-bound HIV protease, and their implications for function. Structure 1999, 7 (9), 1047-55.

(29) Tozzini, V.; Trylska, J.; Chang, C. E.; McCammon, J. A. Flap opening dynamics in HIV-1 protease explored with a coarse-grained model. J. Struct. Biol. 2007, 157 (3), 606-15.

(30) Pietrucci, F.; Marinelli, F.; Carloni, P.; Laio, A. Substrate binding mechanism of HIV-1 protease from explicit-solvent atomistic simulations. J. Am. Chem. Soc. 2009, 131 (33), 11811-8.

(31) Im, W.; Lee, M. S.; Brooks, C. L., III. Generalized born model with a simple smoothing function. J. Comput. Chem. 2003, 24 (14), 1691702.

(32) Brooks, B. R.; et al. CHARMM: The biomolecular simulation program. J. Comput. Chem. 2009, 30 (10), 1545-1614.

(33) Floquet, N.; Marechal, J. D.; Badet-Denisot, M. A.; Robert, C. H.; Dauchez, M.; Perahia, D. Normal mode analysis as a prerequisite for drug design: application to matrix metalloproteinases inhibitors. FEBS Lett. 2006, 580 (22), 5130-6.

(34) Sperandio, O.; Mouawad, L.; Pinto, E.; Villoutreix, B.; Perahia, D.; Miteva, M. How to choose relevant multiple receptor conformations for virtual screening: a test case of Cdk2 and normal mode analysis. Eur. Biophys. J. 2010, 39 (9), 1365-1372.

(35) Floquet, N.; M'Kadmi, C.; Perahia, D.; Gagne, D.; Bergé, G.; Marie, J.; Banères, J.-L.; Galleyrand, J.-C.; Fehrentz, J.-A.; Martinez, J. Activation of the Ghrelin Receptor is Described by a Privileged Collective Motion: A Model for Constitutive and Agonist-induced Activation of a Sub-class A G-Protein Coupled Receptor (GPCR). J. Mol. Biol. 2010, 395 (4), 769-784.

(36) Grossfield, A. WHAM: the weighted histogram analysis method, v.158, http://membrane.urmc.rochester.edu/content/wham (accessed July 2011).

(37) Sadiq, S. K.; De Fabritiis, G. Explicit solvent dynamics and energetics of HIV-1 protease flap opening and closing. Proteins 2010, 78 (14), 2873-85.

(38) Ding, F.; Layten, M.; Simmerling, C. Solution structure of HIV-1 protease flaps probed by comparison of molecular dynamics simulation ensembles and EPR experiments. J. Am. Chem. Soc. 2008, 130 (23), 7184-5.

(39) Kumar, S.; Ma, B.; Tsai, C. J.; Sinha, N.; Nussinov, R. Folding and binding cascades: dynamic landscapes and population shifts. Protein Sci. 2000, 9 (1), 10-9.

(40) Okazaki, K.; Takada, S. Dynamic energy landscape view of coupled binding and protein conformational change: induced-fit versus population-shift mechanisms. Proc. Natl. Acad. Sci. U.S.A. 2008, 105 (32), $11182-7$.

(41) Kar, G.; Keskin, O.; Gursoy, A.; Nussinov, R. Allostery and population shift in drug discovery. Curr. Opin. Pharmacol. 2010, 10 (6), 715-22.

(42) Valente, A. P.; Miyamoto, C. A.; Almeida, F. C. Implications of protein conformational diversity for binding and development of new biological active compounds. Curr. Med. Chem. 2006, 13 (30), 3697-703.

(43) Blackburn, M. E.; Veloro, A. M.; Fanucci, G. E. Monitoring inhibitor-induced conformational population shifts in HIV-1 protease by pulsed EPR spectroscopy. Biochemistry 2009, 48 (37), 8765-7.

(44) Ma, J.; Karplus, M. Ligand-induced conformational changes in ras p21: a normal mode and energy minimization analysis. J. Mol. Biol. 1997, 274 (1), 114-31.

(45) Galiano, L.; Ding, F.; Veloro, A. M.; Blackburn, M. E.; Simmerling, C.; Fanucci, G. E. Drug pressure selected mutations in HIV-1 protease alter flap conformations. J. Am. Chem. Soc. 2009, 131 (2), 430-1. 